\title{
Optimal Life Insurance
}

\author{
by Karl Borch *
}

\section{Introduction}

1.1. A person who wants to arrange his life insurance in the best possible manner will probably be bewildered by the many different offers available from insurance companies. The literature which should guide him through this jungle of offers is usually of little help. Much of it is plain sales talk, although it may be of high technical quality, particularly when it comes to explaining the tax advantages offered by life insurance in different countries. There is certainly literature which seeks to offer unbiased advice, but this does not help much, since the authors and would-be experts seem to disagree among themselves.

1.2. Much of the theoretical literature on life insurance is based on Huebner's concept of "human life value". This is essentially the present value of the future income which will not be realized if the person dies. This is an asset - presumably an intangible one - which, according to Huebner, a rational person should cover by insurance.

This is however controversial. According to Denenberg [2] "Huebner's "human life value" ideas have been endorsed and enshrined by the life insurance texts and prominently featured in the training materials of the American College of Life Underwriters". On the other hand, Josephson [5] states fiatly: "I believe it can be said unequivocally that the Huebner concept did not influence the marketing philosophy of a single life insurance company".

We shall not try to settle this dispute, but we should note that the idea of Huebner has its origin in property insurance. Both authors seem to overlook that the real problem in this field is not to evaluate the property, but to decide if the owner should carry some of the risk himself. Clearly this decision will depend on the cost of insurance cover, and calls for an economic analysis.

1.3. Life insurance has obvious relations to economics, since it is essentially a form of saving. One gives up consumption at the present time, in order to provide for one's own old age, or for dependents one may leave at death. If a person wants to know by how much he shoud cut down current consumption in order to provide better for the future, he will get little help from economic literature. Economic theory is based on "consumer's sovereignty", and leaves it to the individual to decide for himself how he will evaluate current needs in relation to the need for providing for future contingencies. In the theory one does however study how this evaluation is

\footnotetext{
* Professor of insurance economics at the Norwegian School of Economics and Business Administration, Bergen. The author is grateful to Denis Moffet for many heated discussions of this subject.
} 
made, since such information is essential in the construction of general models for predicting saving and investment in the economy as a whole. An economist is more likely to observe, than to preach thrift and frugality with the enthusiasm of a good insurance salesman.

1.4. As life insurance is a form of saving, it will have to compete with other forms of saving. The growing interest in portfolio theory over the last two decades has brought much attention to insurance. Life insurance policies obviously should have a place in an optimal portfolio. How prominent this place should be will clearly depend on the nature of the alternative investments, and this leads to a number of interesting problems, which recently have been studied by several authors, i.a. by Fischer [3] and Richard [7]. We shall not take up these problems in their full generality, and we shall find it convenient to begin our discussion by considering some older models.

\section{The Simplest saving - consumption models}

2.1. The models we shall study go back to the neo-classical schools, and were studied in detail by Marshall [6] and by Fisher [4], who dedicated his book to Böhm-Bawerk [1], "who laid the foundation upon which I have endeavoured to build". The purpose of these studies was to determine the theoretical relationships between savings and interest rates in the economy. The notation used by these writers appears cumbersome to a modern reader, and in the following we shall use a notation which is due to Yaari [8] and [9].

2.2. The given element in the models we shall study is the consumer's income stream $y(t)$, a function of time $t$. The problem is to determine the optimal stream $c(t)$ for his expenditure on consumption. It is convenient to refer to the function $c(t)$ as a consumption plan. Any plan which can be carried out for a given income stream will be called a feasible plan.

If the consumer can neither borrow money, nor save money for future consumption, the feasible consumption plans will be defined by the inequality

$$
c(t) \leqslant y(t)
$$

This simply says that the consumer cannot at any time spend more than his income. Among the feasible plans, the plan $c(t)=y(t)$ will appear as the optimal one, under the usual assumption of non-saturation, i.e. if we assume that the consumer prefers to spend his income on consumption, rather than letting it be wasted.

2.3. In order to arrive at less trivial models, we must assume that the consumer has some possibilities of transfering money from one point of time to another, i.e. that he can reallocate his income over time. The simplest assumption of this kind is that the consumer can borrow or save any amount at the same rate of interest. For an arbitrary consumption plan, his accumulated savings at time $t$ will then be

$$
S(t)=e^{\delta t} \int_{0}^{t} e^{-\delta s}\{y(s)-c(s)\} d s
$$

where $\delta$ is the force of interest. 
It is natural to assume that there are some restriction on the consumer's borrowing. One restriction of this kind would be a condition :

$$
S(T) \geqslant 0
$$

which says that the consumer must be solvent at time $T$. We can interpret $T$ as his "planning horizon" - or that of his creditors.

A stricter condition would be :

$$
S(t) \geqslant 0 \quad \text { for } 0 \leqslant t \leqslant T .
$$

If this condition is imposed, the consumer is never allowed to be in debt, and it is not necessary to assume that interest rates are the same for borrowing and saving.

Either of the conditions (1) or (2) will give a set of feasible consumption plans, and it is evident that (2) will give the more restricted set. If no value is assigned to consumption beyond the horizon, we will have $S(T)=0$ for the optimal plan - under the non-saturation assumptions.

2.4. It is not necessary to assume that $y(t)$ and $c(t)$ are continuous flows. We can define $Y(t)$ and $C(t)$ as accumulated income and consumption up to time $t$. Accumulated savings at time $t$ will then be

$$
S(t)=e^{\delta t}\left\{\int_{0}^{t} e^{-\delta s} d Y(s)-\int_{0}^{t} e^{-\delta s} d C(s)\right\}
$$

There is little to be gained by this trival generalization, and it will not be used in the following discussion.

2.5. To select the optimal plan, we need a preference ordering over the set of feasible consumption plans. It is convenient if this ordering can be represented by a utility functional, i.e. a mapping from the set of feasible plans to the real line, which can be expressed in a simple analytical manner.

In economic theory one usually assumes that the utility assigned to an arbitrary consumption plan is given by an expression of the form :

$$
U(c)=\int_{0}^{T} e^{-\gamma t} u[c(t)] d t .
$$

Here $\gamma$ represents the consumer's "impatience", i.e. his preference for consumption early rather than late in the planning period. For the instantaneous utility function $u(c)$ one usually assumes :

$$
u^{\prime}(c)>0 \quad \text { and } \quad u^{\prime \prime}(c)<0 .
$$

Expression (3) clearly contains a number of "heroic assumptions", and the main merit of models of this type may be that they lead to problems which can be solved by relatively simple mathematical methods. One should however note that it is difficult even to describe other, and possibly more realistic preference orderings over sets of consumption plans.

2.6. We have now arrived at the problem of maximizing (3), subject to some condition, euch as (1) or (2), which will keep the maximand finite. 
From the definition of accumulated savings $S(t)$ we find

$$
S^{\prime}(t)=\delta S(t)+y(t)-c(t)
$$

or

$$
c(t)=y(t)+\delta S(t)-S^{\prime}(t) .
$$

Substituting this in (3) we arrive at the problem of maximizing:

$$
\int_{0}^{T} e^{-\gamma t} u\left[y(t)+\delta S(t)-S^{\prime}(t)\right] d t
$$

This expression is of the form

$$
\int_{0}^{T} F\left[t, x(t), x^{\prime}(t)\right] d t
$$

so the problem is reduced to a problem in the classical calculus of variation. A solution to the problem must satisfy the Euler equation

$$
\frac{\partial F}{\partial x}=\frac{d}{d t}\left(\frac{\partial F}{\partial x^{\prime}}\right)
$$

2.7. In our problem the Euler equation takes the form

which reduces to

$$
\delta e^{-y t} u^{\prime}[c(t)]+\frac{d\left[e^{-y t} u^{\prime}[c(t)]\right]}{d t}=0
$$

$$
\begin{gathered}
c^{\prime}(t)=(\gamma-\delta) \frac{u^{\prime}[c(t)]}{u^{\prime \prime}[c(t)]} \\
u^{\prime}[c(t)]=K e(\gamma-\delta) t
\end{gathered}
$$

Here $K$ is an arbitrary positive constant, which occurs because the solution of the problem remains the same if the utility function is multiplied by a positive constant.

From (4) it follows that $c(t)$ will be constant if $\gamma=\delta$, i.e. if the rate of impatience is equal to the rate of interest. If $\gamma>\delta, c(t)$ will be monotonic decreasing, and if $\gamma<\delta, c(t)$ will be monotonic increasing.

If by pure chance the solution of the problem should be $c(t)=y(t)$, the consumer will neither borrow nor save. In general it will however be to his advantage to transfer income from one period to another.

2.8. It may be useful to give a simple example to illustrate the results above.

Let $u(c)=\log c$. The differential equation (4) then becomes

with the solution

$$
c^{\prime}(t)=(\delta-\gamma) c(t)
$$

$$
c(t)=A e^{(0-\gamma) t} \text {. }
$$

The constant of integration $A=c(0)$ must be determined so that condition (1) is satisfied, with strict equality, i.e. we must have

$$
\int_{0}^{T} e^{-\delta t} y(t) d t=\int_{0}^{T} e^{-\delta t} c(t) d t=A \int_{0}^{T} e^{-\gamma t} d t
$$

Let us further assume $y(t)=y=$ constant for $0 \leqslant t \leqslant T$. 
If in this case $\delta>\gamma$, we will have $c(t)<y$ in the beginning of the period, and $c(t)>y$ towards the end. This plan will be feasible, also if the stricter condition (2) is imposed.

If on the other hand $\delta<\gamma$, we have $c(t)>y$ in the beginning of the period, and this plan is not feasible under condition (2). The optimal feasible plan will then be $c(t)=y$. In this case the introduction of credit facilities would relax the condition (2), and allow the consumer to adopt a better consumption plan.

\section{Models with uncertainty and Insurance}

3.1. In a world of complete certainty there are no compelling reasons for not giving credit to a consumer, provided that his future income is sufficient to repay the debt with interest. If however there is a possibility that the income stream may be cut off at any time, potential creditors may impose the strict condition (2), and not allowing the consumer to be in debt.

To formulate this idea in a simple manner, we shall assume that the income stream $y(t)$ is given, but that it at any time can drop to zero for the rest of the planning period. Let $\pi(t)$ be the probability that the income stream flows at time $t$. We have $\pi(0)=1$.

As a concrete interpretation we can think of situations in which the income ceases because the consumer becomes permanently disabled, or unemployed. The traditional purpose of saving is just to build up a reserve for such events.

3.2. The problem of the consumer must now be reformulated, and it is natural to assume that he will maximize expected utility:

$$
\int_{0}^{T} e^{-v t}\{\pi(t) u[c(t)+y(t)]+[1-\pi(t)] u[c(t)]\} d t
$$

subject to some conditions such as (1) or (2).

As in para 2.6. we can find a differential equation which accumulated savings must satisfy, and solve a problem in the calculus of variation.

This solution to the problem seems inefficient, since it may lead to an accumulation of savings which will not be "needed" if the income stream is maintained until time $T$, i.e. until the end of the planning period. The introduction of insurance will make it possible for the consumer to obtain an income $c(t)$ for consumption if, and only if, his earnings $y(t)$ stops. This possibility may then help the consumer, just as credit facilities did in para 2.8 .

3.3. To make things simple, we shall assume that he hands his whole income $y(t)$ over to an insurance company. In return he receives a stream $c(t)$, which he can use for consumption.

The principle of equivalence implies that we must have

$$
\int_{0}^{T} \pi(t) y(t) e^{-\delta t} d t-\int_{0}^{T} c(t) e^{-\delta t} d t=0 .
$$


This condition which defines the feasible consumption plans, plays the same role as condition (1) in the previous section.

The prospective reserve of the insurance arrangement at time $t$ is :

$$
V(t)=e^{\delta t} \int_{t}^{T} c(s) e^{-\delta s} d s-e^{\delta t} \int_{t}^{T} \pi(s) y(s) e^{-\delta s} d s .
$$

For any insurance contract one usually requires that the reserve shall be nonnegative, i.e. that

$$
V(t) \geqslant 0 .
$$

This is obviously equivalent to condition (2), and the conditions (2) and (7) are usually imposed for similar reasons.

3.4. Insurance has removed the uncertainty, so the problem of the consumer is now

$$
\max \int_{0}^{T} e^{-y t} u[c(t)] d t
$$

subject to condition (6), or to the stricter condition (7).

From the definition of the reserve we find

$$
V^{\prime}(t)=\delta V(t)+\pi(t) y(t)-c(t) .
$$

We can use this to substitute for $c(t)$, and obtain a problem in the calculus of variation.

To compare the two problems (5) and (8), we note that expected income at time $t$ is

$$
E\{y(t)\}=\pi(t) y(t)
$$

Hence the former problem can be written

$$
\max \int_{0}^{r} e^{-\gamma t} E\left\{u\left(\delta S-S^{\prime}+y\right)\right\} d t
$$

and the second

$$
\max \int_{0}^{T} e^{-\gamma t} u\left[\delta V-V^{\prime}+E\{y(t)\}\right] d t .
$$

Formally the two problems are identical, and they will have identical solutions, provided that the stricter conditions (2) and (7) do not become effective.

As we have assumed that $u(c)$ is concave, it follows from Jensen's inequality that we have

$$
E\{u(y)\}<u(E\{y\})
$$

so that problem (8) gives a higher utility than problem (5).

3.5. We have introduced insurance in a rather artificial way. A more conventional way would be to assume that the consumer agrees to pay a stream of premiums $p(t)$ as long as his income flows. In return he receives an income stream $c(t)$ if the stream $y(t)$ should stop. The problem is then to determine an insurance plan which will make it possible to carry out the best possible consumption plan. 
Formally the problem is as before to maximize

$$
\int_{0}^{T} e^{-\gamma t} u[c(t)] d t .
$$

Under this plan accumulated savings at time $t$ will be :

$$
S(t)=e^{\delta t} \int_{0}^{t}\{y(s)-c(s)-p(s)\} e^{-\delta s} d s .
$$

The retrospective reserve under the insurance contract at time $t$ will be :

$$
V(t)=e^{\delta t} \int_{0}^{t} \pi(s) p(s) e^{-\delta s} d s-e^{\delta t} \int_{0}^{t}[1-\pi(s)] c(s) e^{-\delta s} d s .
$$

Differentiating these two equations, and eliminating $p(t)$, we obtain an expression for $c(t)$ which can be substituted into the maximand. The problem then takes a familiar form. If we impose no other conditions on $S(t)$ and $V(t)$ than simple boundary conditions, such as

and

$$
\begin{aligned}
& S(0)=S(T)=0 \\
& V(0)=V(T)=0
\end{aligned}
$$

the problem can be solved by classical calculus of variation.

It can be shown that under the optimal plans $c(t)+p(t)=y(t)$ i.e. $S(t)=0$, so that all saving takes place by building up reserves in the insurance company. This result should not be surprising, since insurance does not lead to any savings which may not be needed.

3.6. The premium is determined by the principle of equivalence, i.e. the equation $V(T)=0$ or $\quad \int_{0}^{T} \pi(t) p(t) e^{-\delta t} d t=\int_{0}^{T}\{1-\pi(t)\} c(t) e^{-\delta t} d t$.

Any function $p(t)$ which satisfies this equation represents a feasible premium plan, although one would usually also require that $V(t) \geqslant 0$.

In conventional insurance contracts the function $p(\mathrm{t})$ is usually of a simple form, for instance $p(t)=p=$ constant. In such cases the difference $y(t)-p(t)-c(t)$ will be saved, or if negative must be covered from accumulated savings. Hence an inflexible plan for paying premiums may force the consumer to save in the conventional way. The point may have some practical interest. Why should a consumer have to put money into the bank in order to pay future "level premiums" to his insurance company?

\section{Life insurance}

4.1. Let us now assume that the income stream ceases only with the consumer's death. If we assume that his need for consumption dies with him, the models in the preceding section do no longer apply. The upper limit $T$ in the integral in the objective function (3), was interpreted as the consumer's planning horizon. His life time is a 
very natural planning horizon indeed, so let us interpret $T$ as the time of the consumer's death. Clearly $T$ is a stochastic variable, and its probability density is

$$
f(T)=\frac{\ell_{x+T}}{\ell_{x}} \mu_{x+T}
$$

Here $\ell_{x}$ is the mortality table, and $\mu_{x}$ is the force of mortality. It is really unnecessary to introduce the symbol $x$, representing the consumer's age, but it is convenient to do so, since this makes it possible to use the standard actuarial notation.

4.2. The expected utility of the life time consumption is then obtained from (3)

$$
\begin{array}{r}
U=\int_{0}^{\infty}\left\{\int_{0}^{T} e^{-\gamma t} u[c(t)] d t\right\} \frac{\ell_{x+T}}{\ell_{x}} \mu_{x+T} d T= \\
\int_{0}^{\infty} e^{-\gamma t} \frac{\ell_{x+t}}{\ell_{x}} u[c(t)] d t .
\end{array}
$$

Hence the problem is to maximize

$$
\int_{0}^{\infty} e^{-y t} \frac{\ell_{x+t}}{\ell_{x}} u[c(t)] d t
$$

subject to some conditions.

The principle of equivalence requires that the expected discounted value of income must be equal to that of the expenditure on consumption, i.e.

$$
\int_{0}^{\infty} e^{-\delta t} \frac{\ell_{x+t}}{\ell_{x}}\{y(t)-c(t)\} d t=0 .
$$

As in para 3.3 we can assume that the consumer agrees to pay his whole income $y(t)$ to an insurance company, and in return receives funds $c(t)$ for consumption. The prospective reserve for this insurance contract is

and we find

$$
V(t)=e^{\delta t} \int_{t}^{\infty} e^{-\delta s} \frac{\ell_{x+s}}{\ell_{x+t}}\{c(s)-y(s)\} d s
$$

$$
V^{\prime}(t)=\left(\delta+\mu_{x+t}\right) V(t)+y(t)-c(t)
$$

It is usual to require that the reserve for an insurance contract must be nonnegative, i.e. that

$$
V(t) \geqslant 0 .
$$

4.3. We have now arrived at the problem of maximizing (9) subject to the condition (12). If we carry out the maximation under only the weaker condition (10), we can determine $c(t)$ from (11), substitute in (9), proceed as in Section 2, and arrive at the same result. We find that the optimal consumption plan is determined by the differential equation

$$
c^{\prime}(t)=(\gamma-\delta) \frac{u^{\prime}[c(t)]}{u^{\prime \prime}[c(t)]}
$$


As $u^{\prime}(c)>0$ and $u^{\prime \prime}(c)<0$ it follows that $c^{\prime}(t)<0$ if $\gamma>\delta$. This is Fisher's case of high impatience. The consumer will be eager for early consumption, and his optimal plan will be represented by a decreasing function $c(t)$.

This is not a reasonable result. It may of course happen that a person has a high consumption early in life, and that his consumption decreases steadily. It is however difficult to accept that a consumption pattern of this form should be the outcome of deliberate lifelong planning. Most people seem to plan for progress, and to improve their lot.

The case $\delta>\gamma$ does not appear much more reasonable, although a person may conceivably plan so that he can enjoy an increasing consumption all through his life.

4.4. To throw more light on the two questions raised above, we shall study a simple special case. Let as in para 2.8. $u(c)=\log c$. The differential equation which determines $c(t)$ then has the solution

$$
c(t)=c(0) e^{(\gamma-\gamma) t} .
$$

Let us further assume that $y(t)=y$ for $0 \leqslant t \leqslant n$, and $y(t)=0$ for $n<t$. This is a case of a typical old age pension.

The initial value $c(0)$ is determined by $(10)$, i.e.

$$
y \int_{0}^{n} e^{-\delta t} \frac{\ell_{x+t}}{\ell_{x}} d t=c(0) \int_{0}^{\infty} e^{-y t} \frac{\ell_{x+t}}{\ell_{x}} d t .
$$

The premium to be paid for this pension plan is

$$
p(t)=y-c(t) \quad \text { for } 0 \leqslant t \leqslant n .
$$

Under condition (12), the plan will not be feasible if $c(0)>y$. In this case it may be optimal to consume the whole income early in life, with good intentions of beginning to pay into a pension plan at some later date.

It is difficult to accept that the consumption plan we have found is superior to the more conventional plan with $c(t)=c=$ constant. The feasible constant consumption level will then be determined by

$$
y \bar{a}_{x: n}=c \bar{a}_{x}
$$

and it will be paid for by the level premium $p=y-c$ up to time $n$.

The paradoxes we have found are due to our rather arbitrary assumptions about the utility function. We shall however not study utility functions which lead to more reasonable results. Instead we shall discuss a more real paradox.

4.5. Let us assume that the consumer has no income in the first part of the planning period. He may then borrow money for consumption, and secure the loan by life insurance. Let $c(t)$ and $p(t)$ be the flow functions representing respectively consumption and premium payment.

At time $t$ the accumulated debt of the consumer will be

$$
S(t)=e^{\delta t} \int_{0}^{t}\{c(s)+p(s)\} e^{-\delta s} d s .
$$


The premium must be sufficient to pay for a life insurance with an amount $S(t)$ payable if the consumer dies at time $t$. The principle of equivalence then gives the relation

$$
\int_{0}^{t} p(s) \frac{\ell_{x+s-\delta s}}{\ell_{x}} d s=\int_{0}^{t} S(s) \frac{\ell_{x+s}}{\ell_{x}} \mu_{x+s} e^{-\delta s} d s .
$$

We see that this equation is satisfied for $p(t)=S(t) \mu_{x+t}$. This corresponds to a pure risk insurance, or a " renewable term" contract. The reserve for this insurance is zero, so that the non-negativity condition (12) is satisfied.

Substituting the expression for $p(t)$ in (13), we obtain

$$
S(t)=e^{\delta t} \int_{0}^{t}\left\{c(s)+\mu_{x+s} S(s)\right\} e^{-\delta s} d s .
$$

Differentiating we obtain the differential equation

which has the solution

$$
S^{\prime}(t)=\left(\delta+\mu_{x+t}\right) S(t)+c(t)
$$

$$
S(t)=\int_{0}^{t} c(s) e^{-\int_{s}^{t}\left(\delta+\mu_{x+r}\right) d r} d s
$$

or in the standard actuarial notation

$$
S(t)=\int_{0}^{t} \frac{D_{x+s}}{D_{x+t}} c(s) d s .
$$

4.6. In the problem discussed above there are no obvious limits neither to $c(t)$ nor to $t$. Hence a person with no prospective income should be able to maintain an arbitrary high level of consumption for any finite period of time, if he is allowed to play the " insurance game" we have outlined.

Apparently nobody loses in the game. The insurance company will receive the premiums, and pay the corresponding amount on the death of the consumer. The lender will receive the loan back, with compound interest when the consumer dies. There are no obvious institutional difficulties. There should for instance be no legal objections if the lender himself takes out life insurance on the consumer, pays the premiums and adds them, with interest to the loan.

Yaari [9] recognizes the paradox, but dismisses it with an assumption that the " company will refuse to issue life insurance after the consumer reaches a certain age". This is correct, but the age limit is in most countries between 70 and 90 , so this is not a very satisfactory explanation. To a man in his early twenties, half a century of no work and unrestrained consumption, even if a day of reckoning is bound to come, may seem an attractive prospect.

4.7. Fisher [4] does not discuss the paradox explicitely, but he recognizes the legitimacy of " consumption loans to anticipate improvement in financial condition". It is however clear from his book that Fisher would have tried to explain the paradox by macroeconomic arguments. Nobody can consume more than his income, unless somebody else is willing to save a part of his income and lend it to the impatient consumer. In 
Fisher's world consumers who want to play the "insurance game" may be unable to find lenders.

This is however not a complete explanation. The interest rate should bring about equilibrium between demand from borrowers and supply from lenders, but it is clear that higher interest would not deter a consumer who wanted to play the game. An increase in the interest rate would just drive out of the market those who want to borrow for investment purposes. Hence life insurance backing for consumer loans must create some inflationary pressure, simply because it makes possible consumption which otherwise would have to be postponed.

The real paradox behind these observations may be that life insurance companies, whose very existence seems to be threatened by inflation, contribute to the inflation by selling term insurance to cover loans which may accelerate consumption. We shall not discuss this question further, since it seems to merit a separate paper. It may however be of interest to mention that Fisher observes that "such loans are made perhaps most often in Great britain" ([4], page 358), a country which has the world's most developed insurance institutions, and also an unenviable rate of inflation.

\section{Insurance for the benefit of survivors}

5.1. We have so far considered life insurance only as a mean to smoothen a fluctuating income stream over an uncertain life time. The solution to the problems considered made certain that the consumer left no unspent savings at his death. Much life insurance is however written for the explicit purpose of leaving liquid assets as "bequests" to heirs. We must therefore conclude that some people assign utility to leaving such bequests.

To bring this element into the model we can assume that the person's consumptioninsurance plan consists of two elements :

(i) a consumption plan for his life time $c(t)$;

(ii) an amount $B(t)$ payable as bequest to the consumer's heirs if he should die at time $t$.

With given resources, i.e. when the income stream $y(t)$ is given, a set of pairs $\{c(t), B(t)\}$ will appear as feasible. The first problem is then to establish a preference ordering over a set of such pairs.

With the assumptions we have made earlier, it is natural to assume that the utility assigned to an arbitrary pair is

$$
\int_{0}^{\infty} e^{-\gamma t} \frac{\ell_{x+t}}{\ell_{x}} u[c(t)] d t+\int_{0}^{\infty} \beta(t) \frac{\ell_{x+t}}{\ell_{x}} \mu_{x+t} w[B(t)] d t .
$$

Here we have written $\beta(\mathrm{t})$ rather than $e^{-\beta t}$, because it seems a little artificial to assume that bequests should be discounted at a constant rate. The function $w(B)$ represents the utility of a bequest $B$. Expression (14) is Yaari's criterion function in standard actuarial notation. 
5.2. In order to determine the feasible pairs, we can again assume that the consumer pays his whole income $y(t)$ to an insurance company, which in return gives him a pair $\{c(t), B(t)\}$. The principle of equivalence then requires that

$$
\int_{0}^{\infty} e^{-\delta t} \frac{\ell_{x+t}}{\ell_{x}} y(t) d t=\int_{0}^{\infty} e^{-\delta t} \frac{\ell_{x+t}}{\ell_{x}}\left\{c(t)+\mu_{x+t} B(t)\right\} d t .
$$

The prospective reserve of this insurance contract is

$$
V(t)=e^{\delta t} \int_{t}^{\infty} e^{-\delta s} \frac{\ell_{x+s}}{\ell_{x+t}}\left\{c(s)+\mu_{x+s} B(s)-y(s)\right\} d s .
$$

From this we obtain

$$
V^{\prime}(t)=\left\{\delta+\mu_{x+t}\right\} V(t)+y(t)-\left\{c(t)+\mu_{x+t} B(t)\right\} .
$$

We can use this to find an expression for $c(t)+\mu_{x+t} B(t)$, and substitute in (14). The problem of maximizing (14) is then reduced to a problem in the classical calculus of variation. The problem becomes more complicated if we impose the natural condition :

$$
V(t) \geqslant 0 \text {. }
$$

5.3. It is not possible to discuss the shape of the solution in any detail, without making some assumptions about the functions $u(c), w(B)$ and $\beta(t)$. It is however not easy to decide which assumptions one should reasonably make, and the literature we have referred to has little to say about this question.

If the purpose of the insurance is to provide income for a surviving widow, it may be natural to put $B(t)=b \bar{a}_{z+t}$, where $z$ is the age of the wife when the insurance arrangement is made. The function $c(t)$ and $b$ must satisfy (15), and be determined so that (14), or some other criterion function is maximized.

This arrangement will give the widow a lump sum, sufficient to buy a life-long annuity for an amount $b$.

5.4. The arrangement we have outlined may be inefficient for two reasons :

(i) if the widow does not want a constant consumption plan, she will after the death of the husband have to solve the problem discussed in Section 4 , and determine her own optimal consumption-insurance plan ;

(ii) the wife may die before the husband, and in this case the bequest will be "wasted" in the same way as the conventional savings discussed in Section 2.

The more general approach would consist in specifying three consumption plans $c_{1}(t), c_{2}(t)$ and $c_{3}(t)$ for respectively the couple, the surviving widow, and the surviving widower. The principle of equivalence will then give the feasible plans, which must satisfy the condition

$$
\int_{0}^{\infty} y(t) \frac{D_{x+t}}{D_{x}} d t=\int_{0}^{\infty}\left\{c_{1}(t)-c_{2}(t)-c_{3}(t)\right\} \frac{D_{x+t, z+t}}{D_{x z}} d t+\int_{0}^{\infty} c_{2}(t) \frac{D_{z+t}}{D_{z}} d t+\int_{0}^{\infty} c_{3}(t) \frac{D_{x+t}}{D_{x}} d t .
$$


The optimal triplet must then be determined so that some criterion function is maximized.

It does not seem realistic to assume that a family shall be able to specify its preferences for future consumption in the form of a criterion function, even more complicated than (14). Such assumptions must however be made by those who design or sell pension plans for group of families. Governmental plans are usually established through a democratic process, and private plans are sold, so we must assume that the plans we find in real life, in some sense are close to optimal. These plans do however differ considerably from one country to another, and from one group to another. If they all are optimal, there must be wide differences in the underlying preferences. If we don't accept this conclusion, we should examine existing plans critically. It may be possible to improve them, without violating the principle of equivalence, which simply says that you pay for what you get.

5.5. To generalize our model, we can consider the family as the unit, and introduce the notation:

$\pi_{s}(t)=$ the probability that the family shall be in state $s$ at time $t$. Here

$$
\sum_{s=1}^{n} \pi_{s}(t)=1
$$

$y_{s}(t)=$ the family's income at time $t$, if it is in state $s$.

$c_{s}(t)=$ the family's consumption at time $t$, if it is in state $\mathrm{s}$.

The principle of equivalence then gives

$$
\sum_{s=1}^{n} \int_{0}^{\infty} e^{-s t} \pi_{s}(t)\left\{y_{s}(t)-c_{s}(t)\right\} d t=0
$$

This equation gives the set of feasible consumption plans $c_{s}(t)$ for the different states. The given elements are the income streams $y_{s}(t)$ and the state probabilities $\pi_{s}(t)$. In order to determine the optimal plan, we need information about the family's preferences, and this may be difficult to obtain in an articulate form.

If the purpose of life insurance is to provide income for the family in different states, there is no need for the conventional insurance contract with a lump sum payable at death - or more generally - when the family makes a transition from one state to another. This contract is however flexible in the sense that it makes it possible for the family to readjust its consumption plan after a change of state. The flexibility may be worth some theoretical loss in efficiency, at least to the normal family unable to specify a complete preference ordering over future consumption in all possible states. 


\section{REFERENCES}

1. BÖHM-BAWERK, E., Positive Theorie des Kapitals, Vienna, 1889.

2. DENENBERG, H.S., “Author's Reply", The Journal of Risk and Insurance, 1970, 648-654.

3. FISCHER, S., "A Life Cycle Model of Life Insurance Purchasing", International Economic Review, 1973, 132-152.

4. FISHER, I., The Theory of Interest, Macmillan, 1930.

5. JOSEPHSON, H.D., "A New Concept of the Economics of Life Value and the Human Life Value : Comment", The Journal of Risk and Insurance, 1970, 641-643.

6. MARSHALL, A., Principles of Economics, Macmillan, 1890.

7. RICHARD, S. F., "Optimal Consumption, Portfolio and Life Insurance. Rules for an Uncertain Lived Individual in a Continuous Time Model", Journal of Financial Economics, 1975, 187-203.

8. YAARI, M.-E., “ On the Existence of an Optimal Plan in a Continuous Time Allocation Process ", Econometrica, 1964, 576-590.

9. YAARI, M. E., “Uncertain Lifetime, Life Insurance, and the Theory of the Consumer", Review of Economic Studies, 1965, 137-150. 